\title{
Continuous Summative Assessment Sessions in Vocational STEM Education
}

\author{
Gordan Durovic \\ University of Rijeka \\ Rijeka, Croatia \\ gdurovic@uniri.hr
}

\author{
Martina Holenko Dlab \\ University of Rijeka \\ Department of Informatics \\ Rijeka, Croatia \\ mholenko@inf.uniri.hr
}

\author{
Natasa Hoic-Bozic \\ University of Rijeka \\ Department of Informatics \\ Rijeka, Croatia \\ natasah@inf.uniri.hr
}

\begin{abstract}
Continuous approach to learning is essential for mastering course content in STEM education. Research results regarding students' learning habits show that students usually learn in a non-continuous way, adopting surface instead of a deep approach to learning. Lack of students' motivation was identified as one of the causes that contribute to the observed problem. In order to motivate students for continuous learning, weekly organized summative assessment sessions were introduced for vocational STEM students in higher education. These sessions were conducted throughout the semester within a typical STEM course. Obtained research results showed that this approach to summative assessment encouraged the majority of students to start to learn continuously. Also, students were motivated to solve more math-based tasks during the preparation for the exam than they would usually do. Encouraging students to learn in a continuous way affected their motivation, thus helping them to move from the surface toward a deep approach to learning.
\end{abstract}

Keywords-ELARS, formative assessment, continuous summative assessment, STEM, online evaluation system 